Revue des patrimoines

36 | 2018

Les archives photographiques de presse, pratiques comparées et enjeux méthodologiques

\title{
Constitution d'une collection, pratiques, circuit des tirages photographiques et conventionnement, le point de vue d'une agence photographique historique, Roger-Viollet (1938-2005)
}

The making of a photo collection: practices, prints and contracts at a historic photography agency, Roger-Viollet (1938-2005)

Delphine Desveaux

\section{(2) OpenEdition}

1 Journals

\section{Édition électronique}

URL : http://journals.openedition.org/insitu/18093

DOI : 10.4000/insitu.18093

ISSN : 1630-7305

Éditeur

Ministère de la culture

Référence électronique

Delphine Desveaux, «Constitution d'une collection, pratiques, circuit des tirages photographiques et conventionnement, le point de vue d'une agence photographique historique, Roger-Viollet (1938-2005) », In Situ [En ligne], 36 | 2018, mis en ligne le 15 octobre 2018, consulté le 03 mai 2019. URL : http:// journals.openedition.org/insitu/18093 ; DOI : 10.4000/insitu.18093

Ce document a été généré automatiquement le 3 mai 2019.

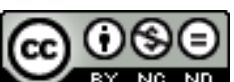

In Situ Revues des patrimoines est mis à disposition selon les termes de la licence Creative Commons Attribution - Pas d'Utilisation Commerciale - Pas de Modification 4.0 International. 


\section{Constitution d'une collection, pratiques, circuit des tirages photographiques et} conventionnement, le point de vue d'une agence photographique historique, Roger-Viollet
(1938-2005)

The making of a photo collection: practices, prints and contracts at a historic photography agency, Roger-Viollet (1938-2005)

Delphine Desveaux

La Documentation photographique Roger-Viollet, créée en 1938, agence de presse depuis 1963, est devenue avec son legs à la Ville de Paris, en 1986, une collection patrimoniale, constituée d'une multitude de fonds. Elle a rejoint au 1er janvier 2018 la Bibliothèque historique de

la Ville de Paris (BHVP) sous forme de département.

Fondé en 1938 par Hélène Roger et Jean (Hans) Fischer ${ }^{1}$, Roger-Viollet s'installe au 6 rue de Seine dans les locaux, les meubles et les photographies de Laurent Ollivier, «marchand d'images» reconnu depuis 1880. À l'époque, l'emplacement de la boutique ollivier répondait de manière opportune à son environnement où se côtoyaient étudiants, artistes, revues et maisons d'édition, autant de clients potentiels en quête de modèles d'art à copier, d'inspiration et d'illustrations (fig. 1, fig. 2). 
Figure 1

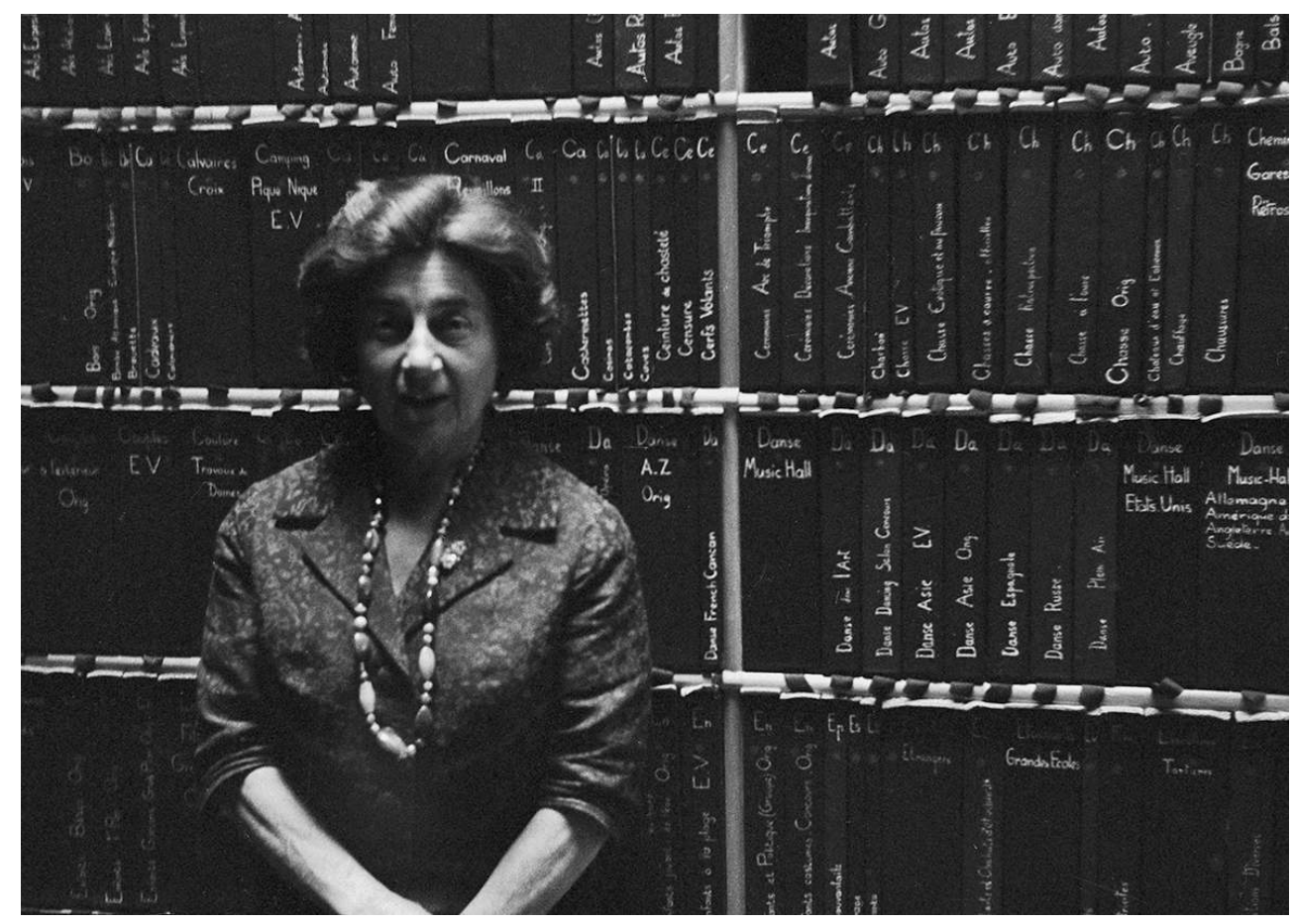

Hélène Roger, vers 1965.

(c) Jean Fischer / Roger-Viollet.

Figure 2

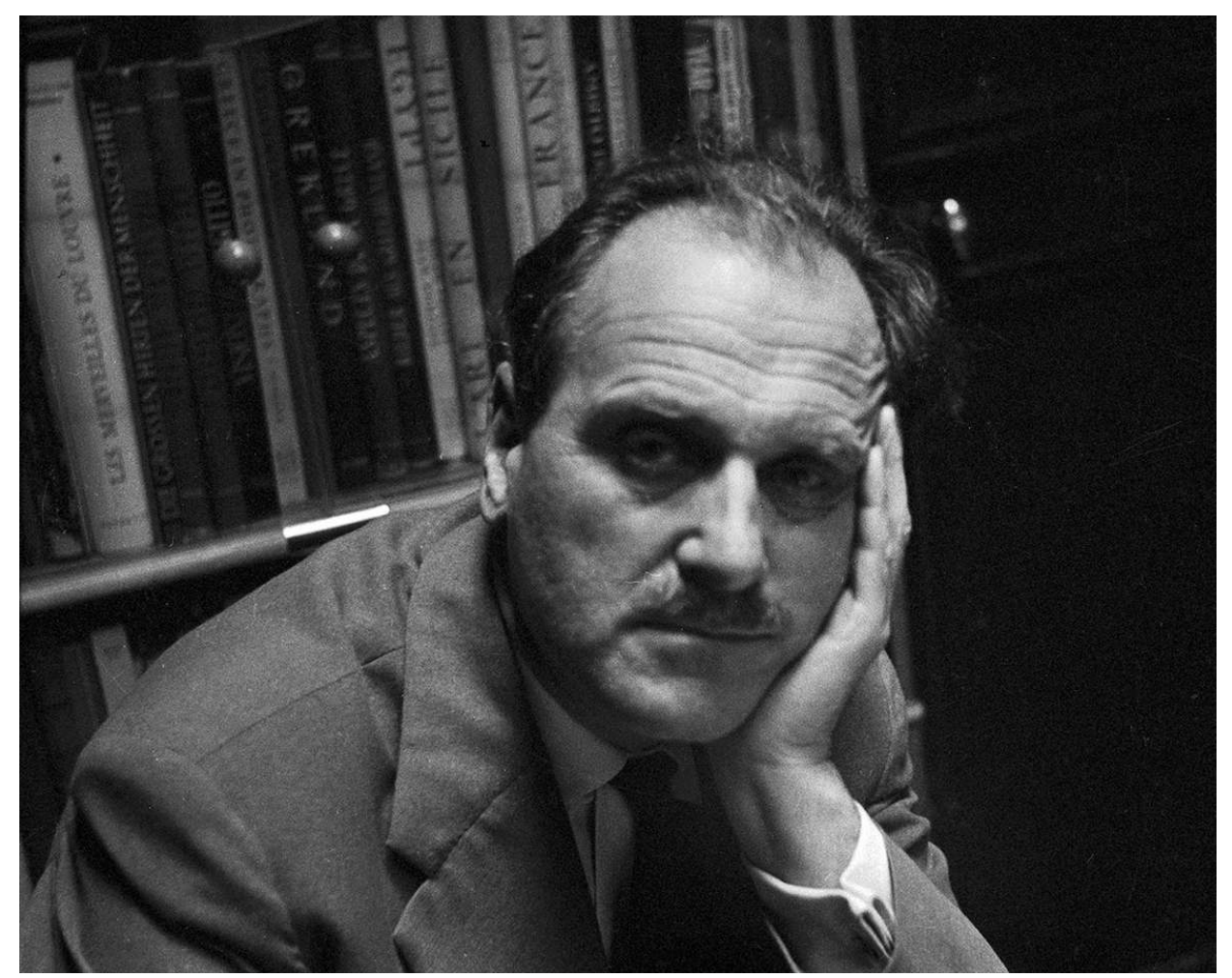

Jean Fischer vers 1965.

(c) Hélène Roger / Roger-Viollet. 
2 Laurent Ollivier avait déjà, en 1913, racheté le fonds d'un imprimeur photographe, Léopold Mercier², constituant ce faisant une offre iconographique de quelque 50000 photographies.

3 Les Collections Roger-Viollet sont montées à partir de trois beaux ensembles : les fonds Ollivier et Mercier déjà cités, auxquels Hélène Roger adjoint la collection personnelle de sa famille, riche déjà, elle aussi, de quelque 50000 phototypes transparents en 1938.

4 Les familles Roger et Viollet sont en effet de ces familles où les amateurs de photographie ne manquaient pas. Citons les plus importants, Henri Roger (1869-1946), le père d'Hélène, ingénieur de formation, inventeur et marchand de bronzes d'art pour faire vivre son épouse et ses six enfants; Ernest Roger (1864-1943), frère aîné d'Henri, ingénieur également et l'un des inventeurs de la télégraphie sans fil avec Eugène Ducretet; Henri (dit Henry) Viollet (1880-1955), frère de Jeanne Viollet, la mère d'Hélène, architecte et archéologue, spécialiste de l'Iran où il multiplia fouilles et voyages ${ }^{3}$ (fig. 3, fig. 4).

Figure 3

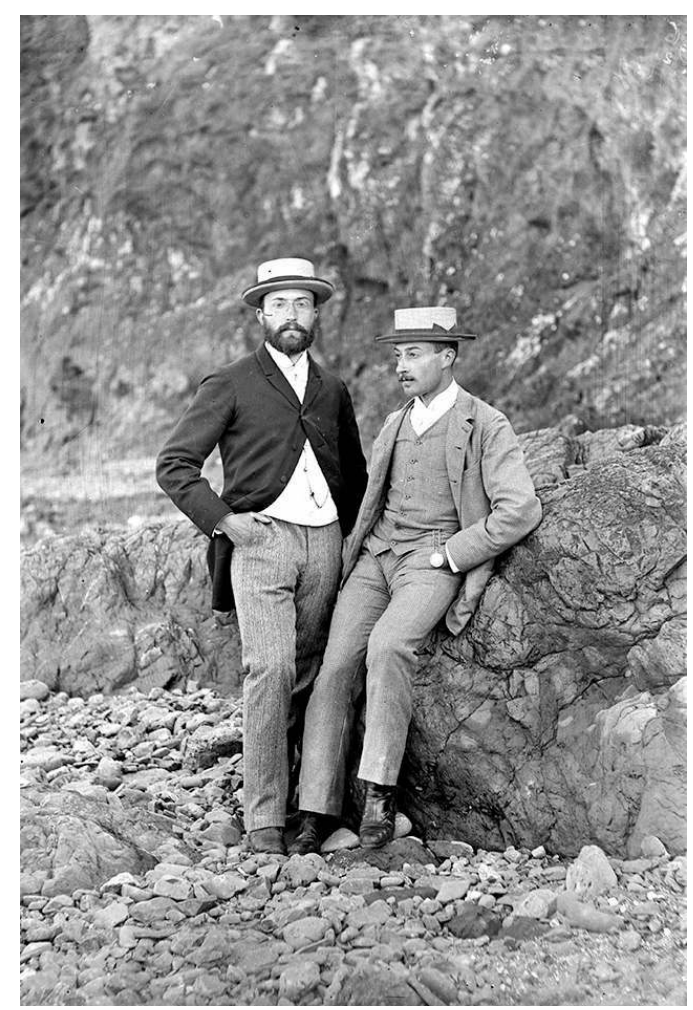

Ernest et Henri Roger sur la plage de Granville, Manche, 1892.

(c) Anonyme / Roger-Viollet. 
Figure 4

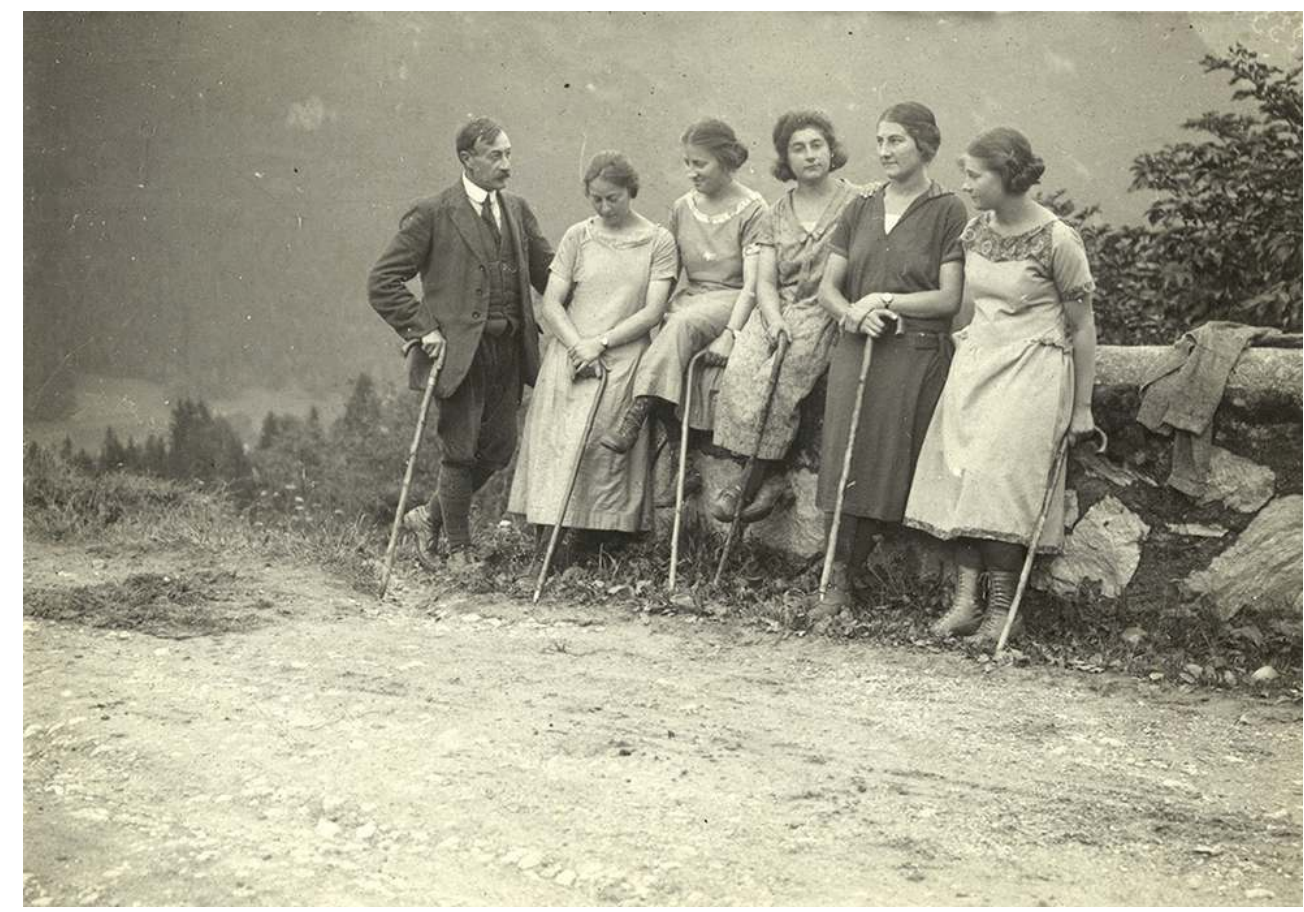

Henri Roger et ses filles. Au centre, Hélène Roger. France, vers 1925.

(c) Henri Roger / Roger-Viollet.

5 Repliés à Pierrelatte, dans la Drôme, pendant la Seconde Guerre mondiale, Hélène Roger et Jean Fischer y inventent une fabrique de savon, dentifrice et lessive, toute une gamme de produits composés à partir de la terre argileuse du pays dont les vertus nettoyantes avaient été redécouvertes par hasard lors d'une lessive improvisée dans une rivière par Hélène Roger... Ce « détour régional » démontre, s'il en était besoin, que les fondateurs de l'agence avaient sans aucun doute ce que l'on nomme la bosse du commerce : toujours en éveil, attentifs (voire opportunistes, parait-il), ils surent durant toute leur carrière à la fois tenir boutique et enrichir leur offre commerciale, donc les Collections Roger-Viollet.

6 De retour à Paris en 1944, le couple a l'agréable surprise de découvrir que le rideau baissé de leur devanture du 6 rue de Seine a préservé leur commerce de tout pillage et, à partir de 1945, il se lance dans une course effrénée d'acquisitions: fonds de studios photographiques, fonds de photographes professionnels, fonds de photographes amateurs, périodiques illustrés, arts graphiques, bibliophilie... (fig. 5, fig. 6) 
Figure 5

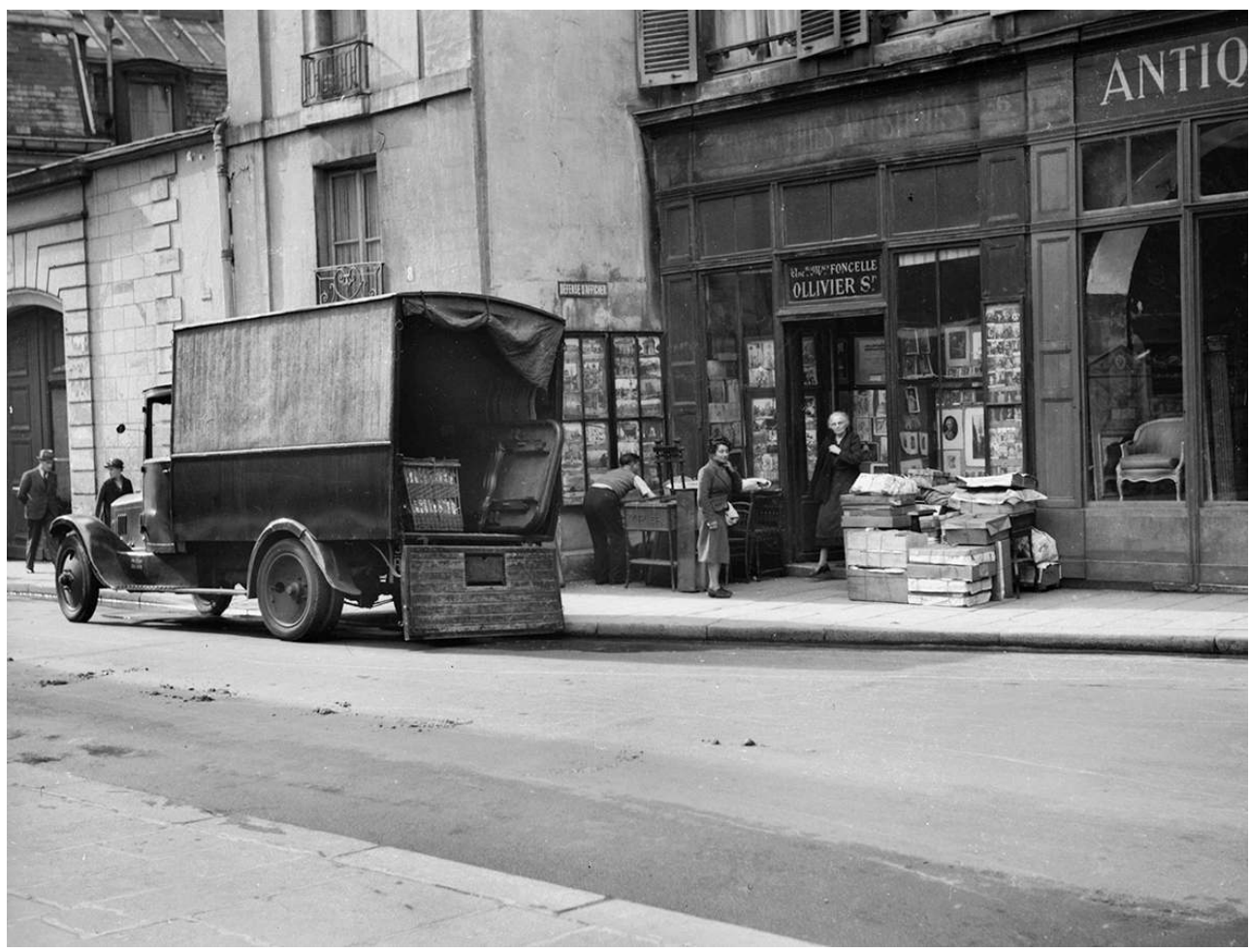

Installation de Roger-Viollet et départ des Ollivier du 6 rue de Seine, 14 octobre 1938.

(C) Jean Fischer / Roger-Viollet. 
Figure 6

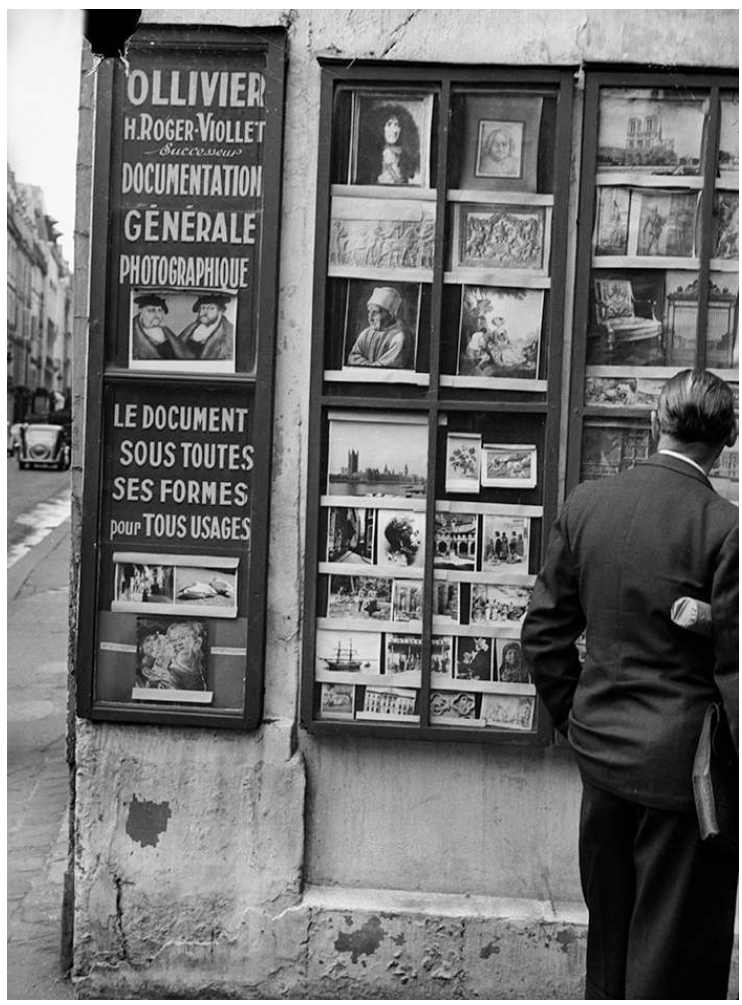

Une des vitrines du 6 rue de Seine, vers 1940.

(c) Hélène Roger-Jean Fischer / Roger-Viollet.

7 Ensembles pléthoriques ou support à la pièce, $\mathrm{XVII}^{\mathrm{e}}, \mathrm{XVIII}^{\mathrm{e}}$ et évidemment $\mathrm{XIX}^{\mathrm{e}}$ et $\mathrm{XX}^{\mathrm{e}}$ siècle puisque la photographie est leur cœur de métier, tout ce qui est illustré «y passe » ainsi que du petit mobilier, des tableaux... et une statue de la Vierge. Et ce pour la bonne raison que le but des fondateurs de cette Documentation photographique Roger-Viollet est de réunir en un seul lieu de quoi répondre à tous les besoins iconographiques possibles et imaginables. Ils composent, en quelque sorte, le pendant du grand magasin de l'autre côté de la Seine, une Samaritaine de la photographie ! (fig. 7)

\section{Figure 7}

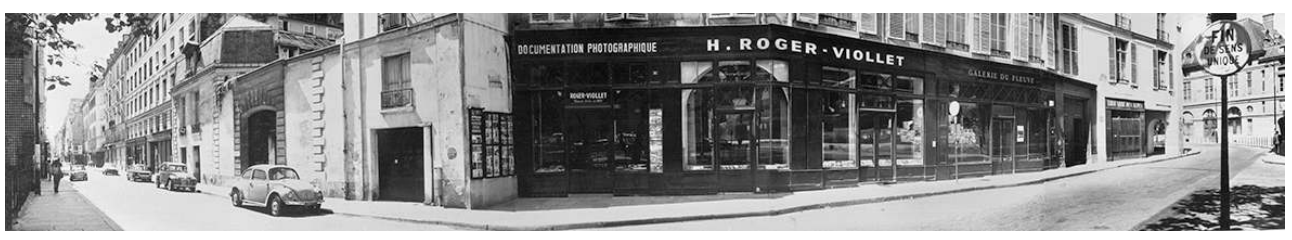

Panoramique du site Roger-Viollet au 6 rue de Seine, vers 1968.

(c) Anonyme / Roger-Viollet.

\section{Constitution des Collections Roger-Viollet}

8 Hélène Roger et Jean Fischer décident, dès l'acquisition du 6 rue de Seine, de ne pas suivre la voie (classique alors) de Laurent Ollivier qui vendait les tirages présents dans son magasin comme il l'aurait fait de n'importe quelle gravure ou estampe. Hélène, qui avait 
appris la photographie et les techniques de laboratoire avec son père, était sensible, en bonne fille d'inventeur, à la propriété intellectuelle. Aussi décident-ils de prêter les tirages et de ne céder que des droits de reproduction en fonction des utilisations qu'en feraient les clients; l'usage est en effet de prêter le support de l'image, le tirage photographique, le client reproduisant l'image sur le support de son choix, et rendant après utilisation ce tirage de presse à l'agence de provenance, laquelle le remet en circulation, s'épargnant de ce fait, la dépense d'en réaliser un grand nombre. En ce sens, et dès 1945, quand ils font l'acquisition de fonds, les Roger-Fischer négocient systématiquement avec le photographe ou ses ayants droit l'acquisition conjointe des droits d'auteur afin de disposer d'une liberté complète d'action dans l'exercice de leurs activités commerciales.

9 Ainsi, les fonds Alain Adler, Laure Albin Guillot, Arlequin, Roger Berson, Jacques Boyer, Maurice-Louis Branger, Tony Burnand, Pierre Choumoff, Compagnie des Arts photomécaniques, Robert Delhay et l'agence LAPI, Ferrier-Soulier, Ray Halin, Albert Harlingue, Marcelle d'Heilly, Charles Hurault, Léon \& Lévy, Boris, Studio et Bernard Lipnitzki, Henri et Élysée Martinie, Neurdein, Gaston Paris, Oswald Perrelle, Georges Pillement, Claude Poirier, Rapoport (Photos Rap), Martin Rikli, Geneviève Vanhaecke... entre autres (mais pour les plus importants) entrent dans l'escarcelle d'Hélène Roger et de Jean Fischer entre 1945 et 1985.

Ces acquisitions toutes françaises et essentiellement parisiennes (quand bien même leurs contenus sont internationaux en termes de sujets) sont complétées par la production du couple, oiseaux migrateurs et voyageurs dans l'âme, qui passe une grande partie de l'année à parcourir le globe afin de documenter le monde et offrir dans les fameuses boîtes vertes ce qui n'y est pas encore (fig. 8, 9, 10). 
Figure 8

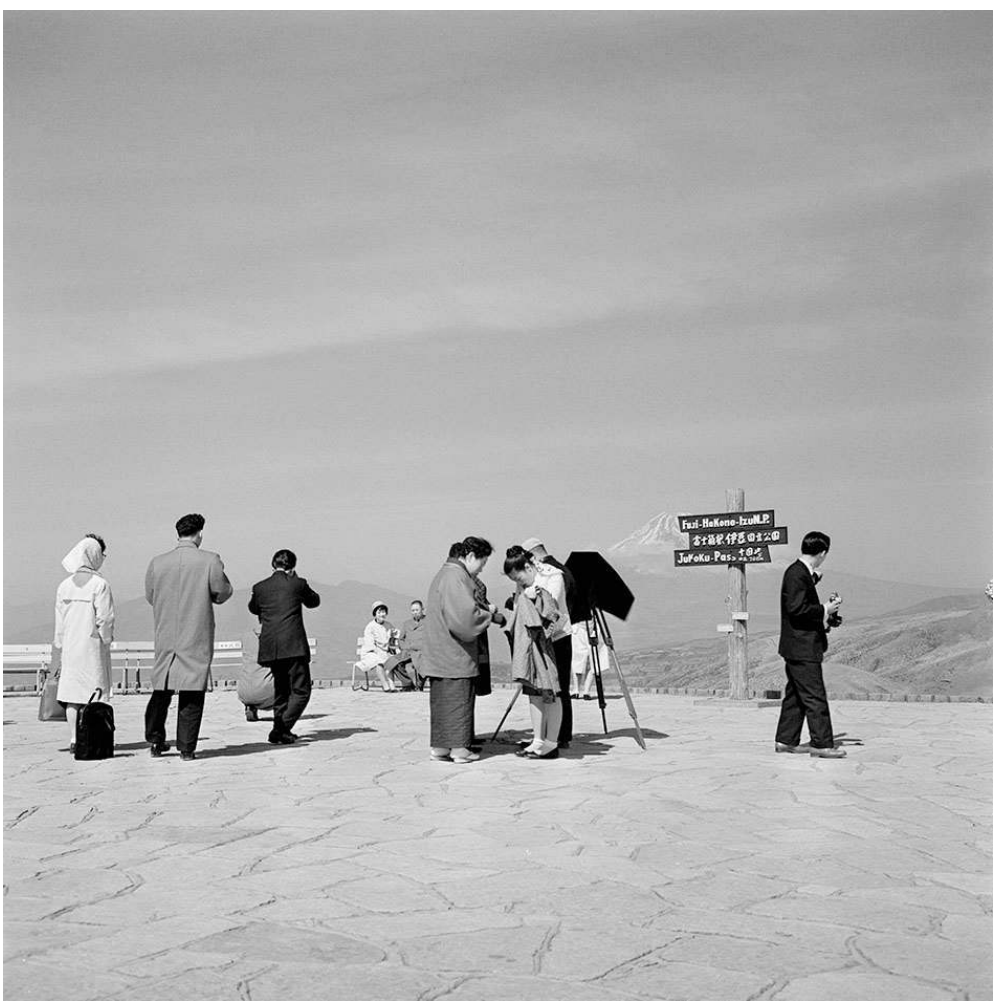

Touristes au mont Jikkoku-Toge (766 m). Au fond, le Fuji-Yama. Japon, mars 1962.

(c) Hélène Roger / Roger-Viollet. 
Figure 9

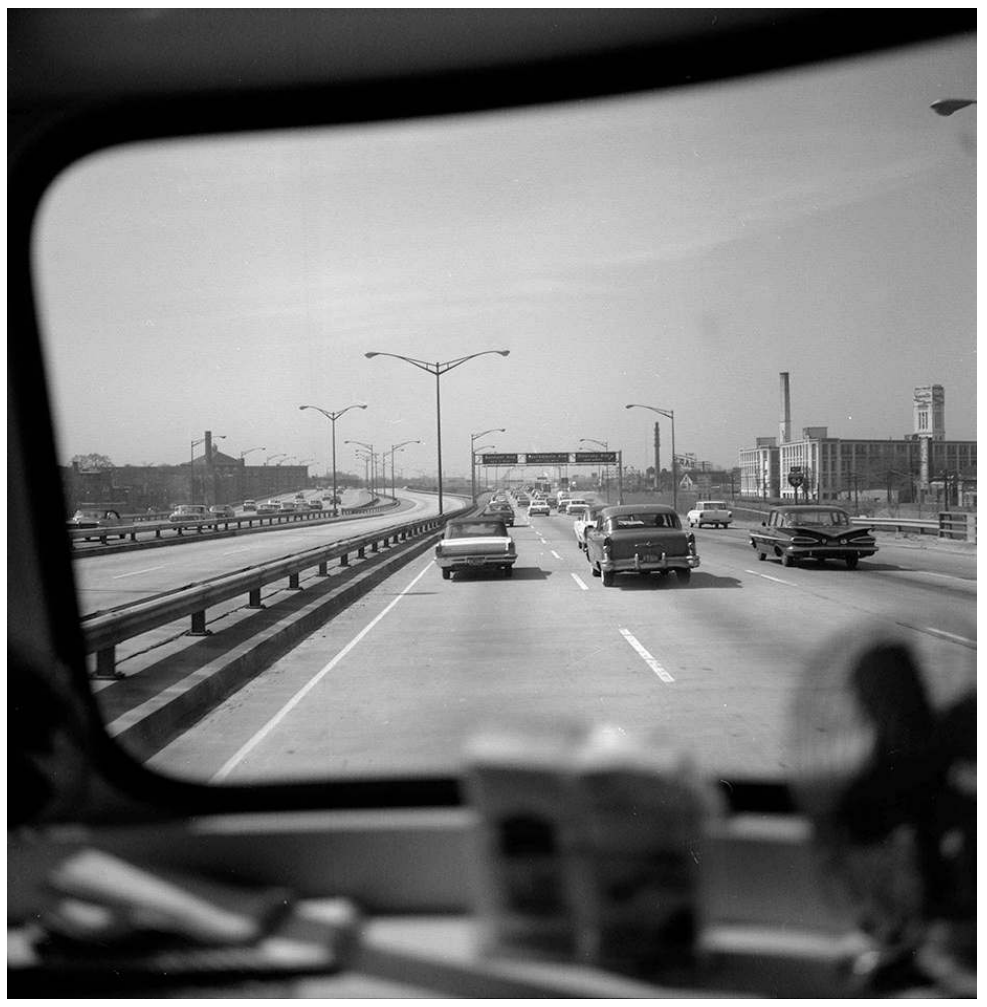

L'autoroute vers l'aéroport de Chicago, États-Unis, 1964.

(c) Hélène Roger-Jean Fischer / Roger-Viollet. 
Figure 10

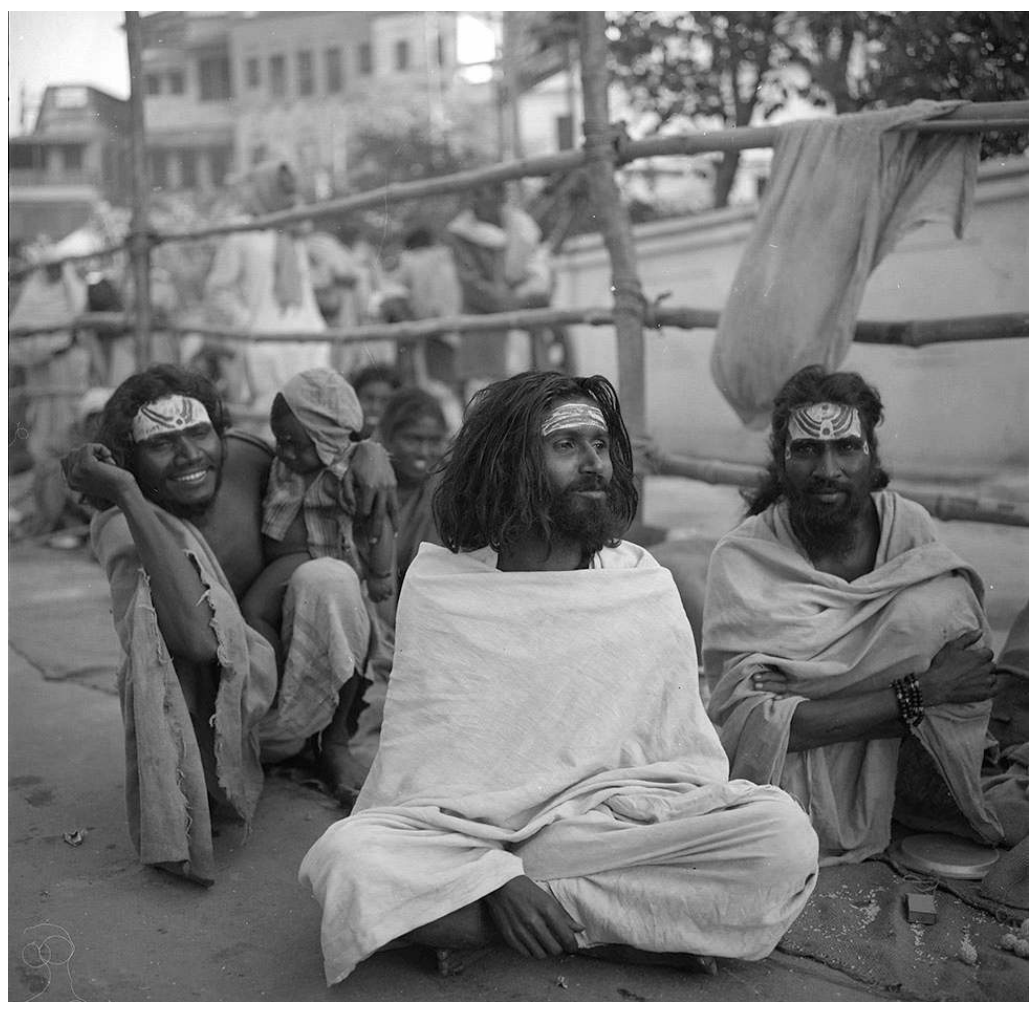

Pèlerins. Bénarès, Uttar Pradesh, Inde, 1961.

(c) Hélène Roger-Jean Fischer / Roger-Viollet.

11 Ceci pour les ensembles homogènes puisque, comme presque toujours en photographie, les reproductions d'œuvres d'autres auteurs existent chez chaque photographe, et a fortiori dans les agences de presse ${ }^{4}$. Il est à noter également que les acquisitions à la pièce ou en petits lots ont été très nombreuses, composant un répertoire formidable de l'histoire de la photographie (ainsi qu'un répertoire formidable de chausse-trappes en termes d'exploitation!).

12 En cours d'inventaire depuis 2002, les quelque 4 millions de phototypes transparents, 2 millions de tirages d'époque et 2 millions de tirages plus modernes ainsi que les quelque 6000 albums photographiques, les photographies enchâssées et les cartes postales, par dizaines de milliers, requièrent encore un certain temps d'analyse... (fig. 11, fig. 12) 
Figure 11

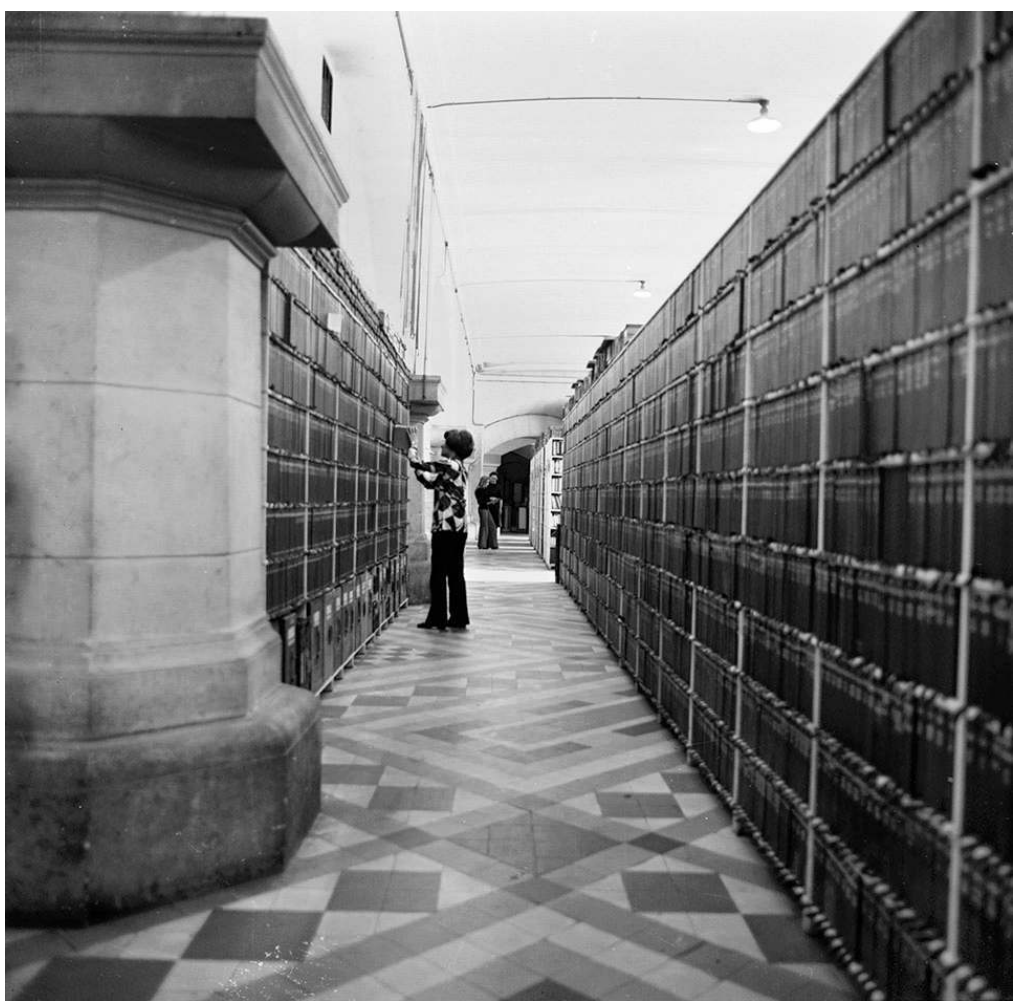

Collections Roger-Viollet, entresol du Petit Palais, musée des Beaux-Arts de la Ville de Paris, 1980 (C) Anonyme / Roger-Viollet. 
Figure 12

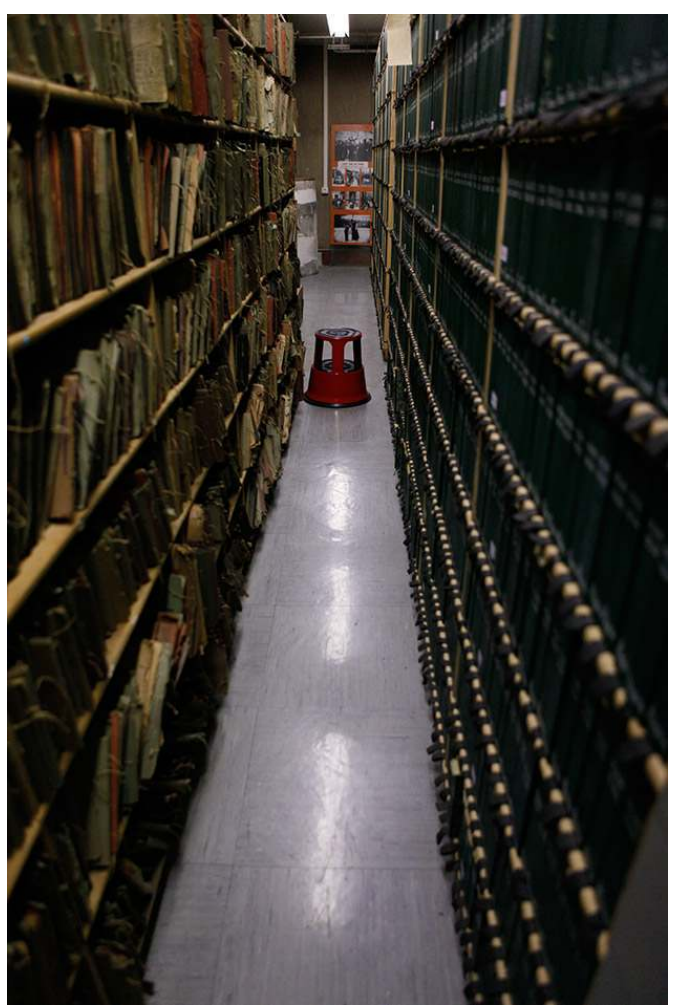

Collections Roger-Viollet; à gauche, albums de la Compagnie des Arts photomécaniques, à droite, fonds Ferrier-Soulier, rue des Arquebusiers, 2011.

(c) Denis Fruit / Parisienne de Photographie. composent de grands ensembles (histoire, géographie, beaux-arts, sports, mode, spectacles, sciences, affaires criminelles, vie militaire, etc.). À l'intérieur des boîtes, des pochettes en papier kraft très résistant en détaillent aussi finement que possible le contenu afin de réduire le champ et le temps de recherche (rentabilité oblige). Chaque tirage porte au verso le numéro de son négatif pour que l'on puisse procéder rapidement à des retirages si besoin. Les négatifs de grands et moyens formats sont aussi classés sur chant dans des boîtes vertes, et ce autant pour les plaques de verre que pour les supports souples, les négatifs de petits formats étant, eux, rangés dans des tiroirs ${ }^{5}$. historiques, choisis avec soin pour leur culture générale et leurs connaissances historiques et géographiques ${ }^{6}$, étant à même d'identifier quasiment toutes les personnes et les sites figurant sur les reproductions d'œuvres d'art et sur les photographies. De 1945 à 1995, ces documentalistes conjuguent leurs activités commerciales avec une politique intense d'identification, de légendage et de corrélation entre les phototypes transparents et les tirages. 


\section{de négatifs sont ainsi montées et descendues, les supports faisant l'objet de rangements précaires en début d'analyse du fonds puis définitifs quand ils avaient trouvé leur destination, thématique pour les tirages, par format pour les phototypes transparents. Les classements thématiques étaient en perpétuelle évolution puisque quand une demande iconographique générait la consultation de trop de boîtes, handicapant l'iconographe en lui prenant trop de temps, une nouvelle boite verte était créée par les documentalistes pour aller dans le sens des besoins de la clientèle. \\ En ce sens, les Collections Roger-Viollet, classées sans recours à un thésaurus mais suivant une logique intellectuelle et commerciale imparable, ont évolué de manière organique en suivant les demandes et évolutions de la profession d'iconographe. Le plan de classement, d'une finesse merveilleuse, a fait la force de l'agence par son efficacité quand les phototypes transparents ont réussi à «tenir » dans les locaux tant ils avaient bénéficié d'une ergonomique économie d'espace (fig. 13, fig. 14).}

l'intégrations toute agence ou instance reprenant des fonds photographiques,

Figure 13

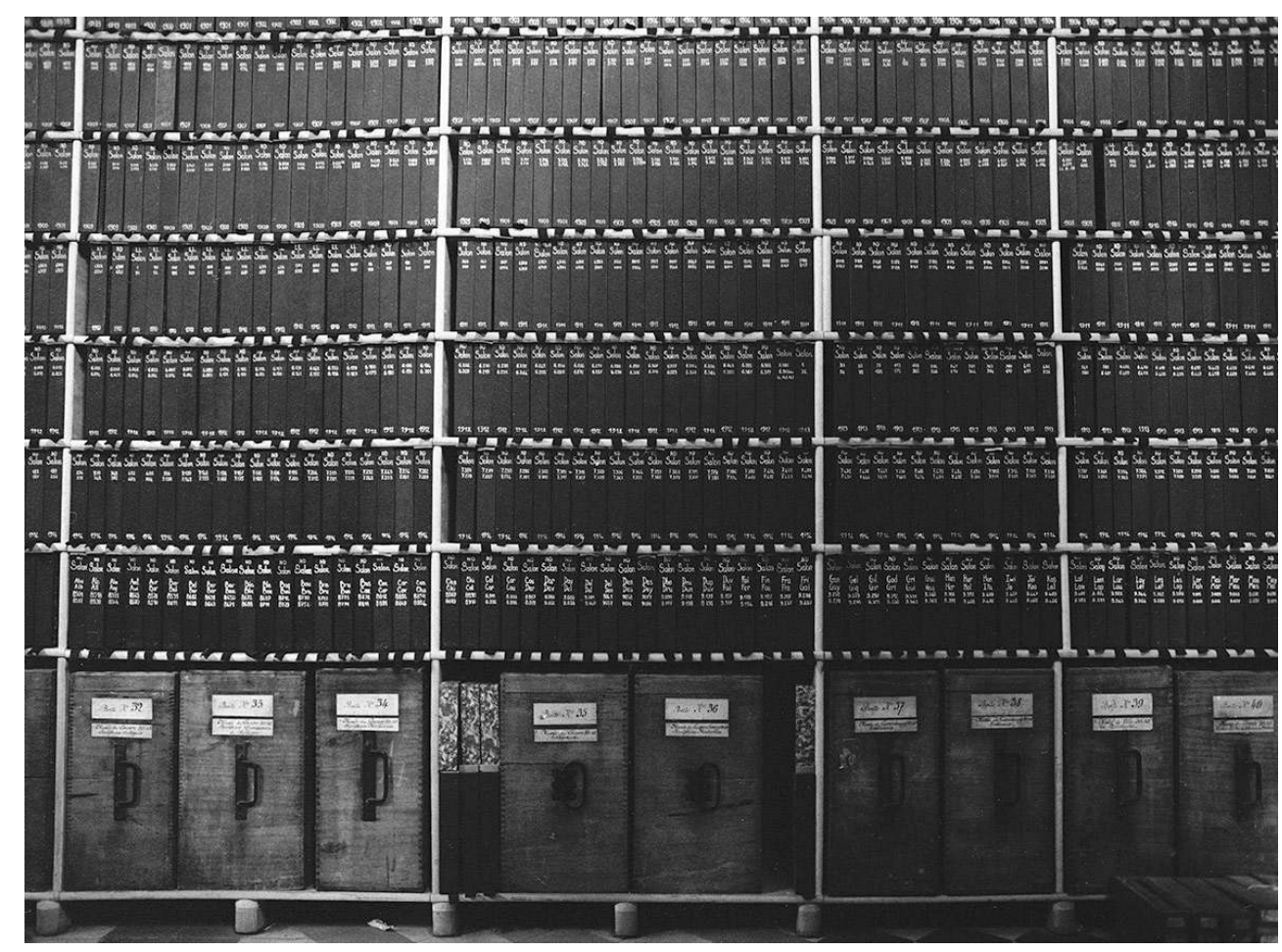

Collections Roger-Viollet, entresol du Petit Palais, musée des Beaux-Arts de la Ville de Paris, 1980. (c) Anonyme / Roger-Viollet. 
Figure 14

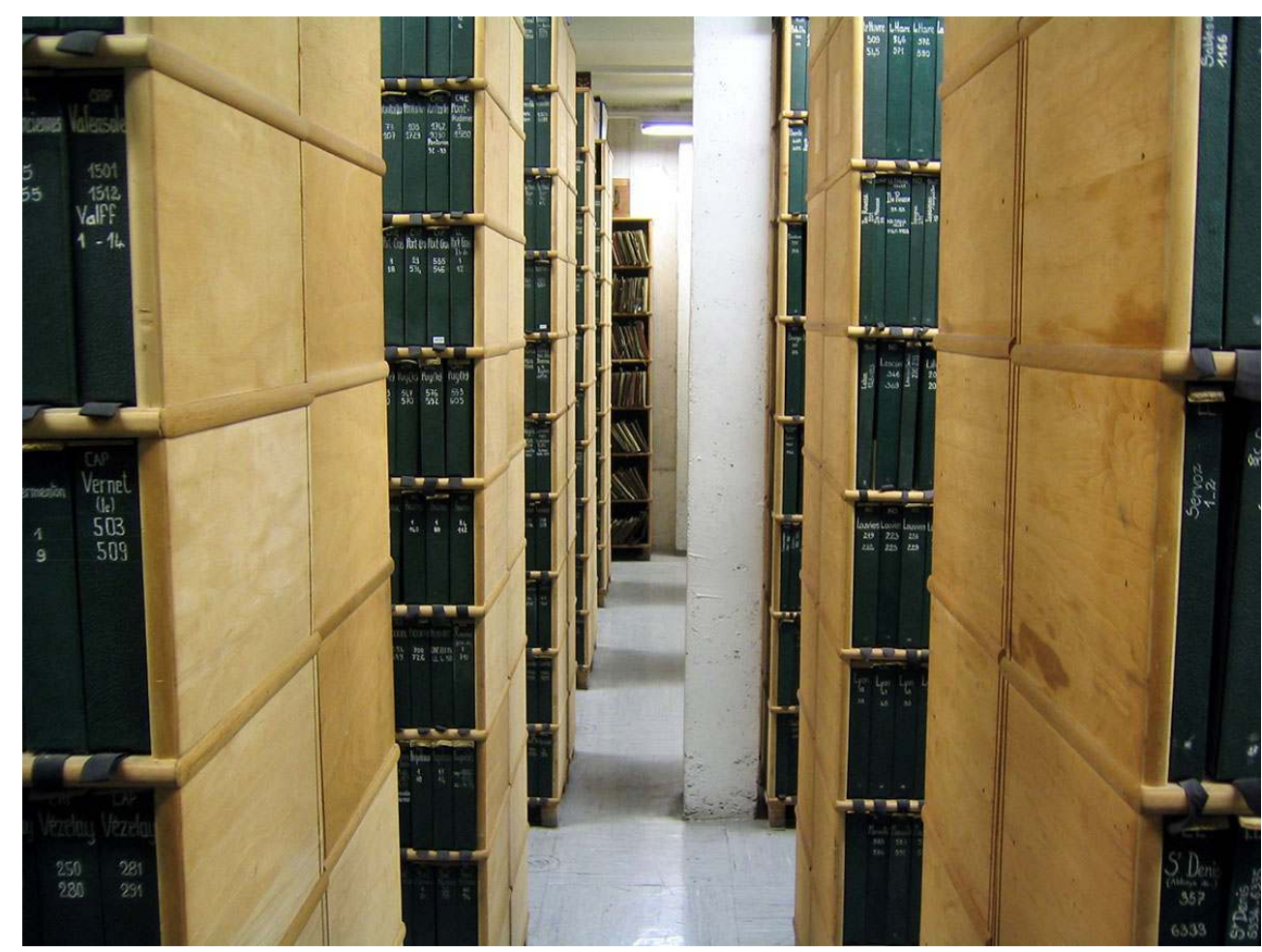

Collections Roger-Viollet, les fonds Ferrier-Soulier, Neurdein, Léon \& Lévy, Compagnie des Arts photomécaniques, rue des Arquebusiers, 2011.

(C) Denis Fruit / Parisienne de Photographie.

Pléthoriques, les Collections Roger-Viollet étaient encore conservées fin 2017 sur cinq sites: 6 rue de Seine, 3 rue des Arquebusiers, 7 rue Dubrunfaut, une cellule au Centre national de la cinématographie ( $\mathrm{CNC}$ ) et des mètres linéaires chez un stockeur en province. Il va sans dire que, excepté les supports qui exigent des conditions de conservation spécifique (climat froid par exemple), les Collections Roger-Viollet ont vocation à être rassemblées sur un site unique.

\section{Fonctionnement de l'agence Roger-Viollet}

19 À de rares exceptions près, le principe qui régit la diffusion des tirages de presse est que leur prêt est assorti d'une obligation de restitution : les tirages Roger-Viollet étaient, à cette fin, tamponnés au verso et portaient une légende (manuscrite puis tapuscrite) ainsi que le numéro du négatif à partir duquel ils avaient été obtenus. Il est à noter qu'entre 1985 et 1995, au vu de la politique intense de tirages des quarante années précédentes et de l'insistance des documentalistes à obtenir le retour des tirages confiés (assortis de frais de traitement pour de grande quantité de tirages sortis sans utilisation), assez peu de tirages modernes ont été réalisés.

20 Une méthode ferme qui laisse des souvenirs piquants à nombre de documentalistes et iconographes, il faut l'avouer, mais encore une fois, une méthode efficace et rentable. Les tirages anciens pouvaient (rarement, heureusement, et dûment tamponnés «à retourner ») être prêtés en urgence à des clients de confiance mais jamais vendus, la 
politique étant que seules les reproductions récentes faisaient l'objet des allers-retours agence-client (fig. 15).

\section{Figure 15}

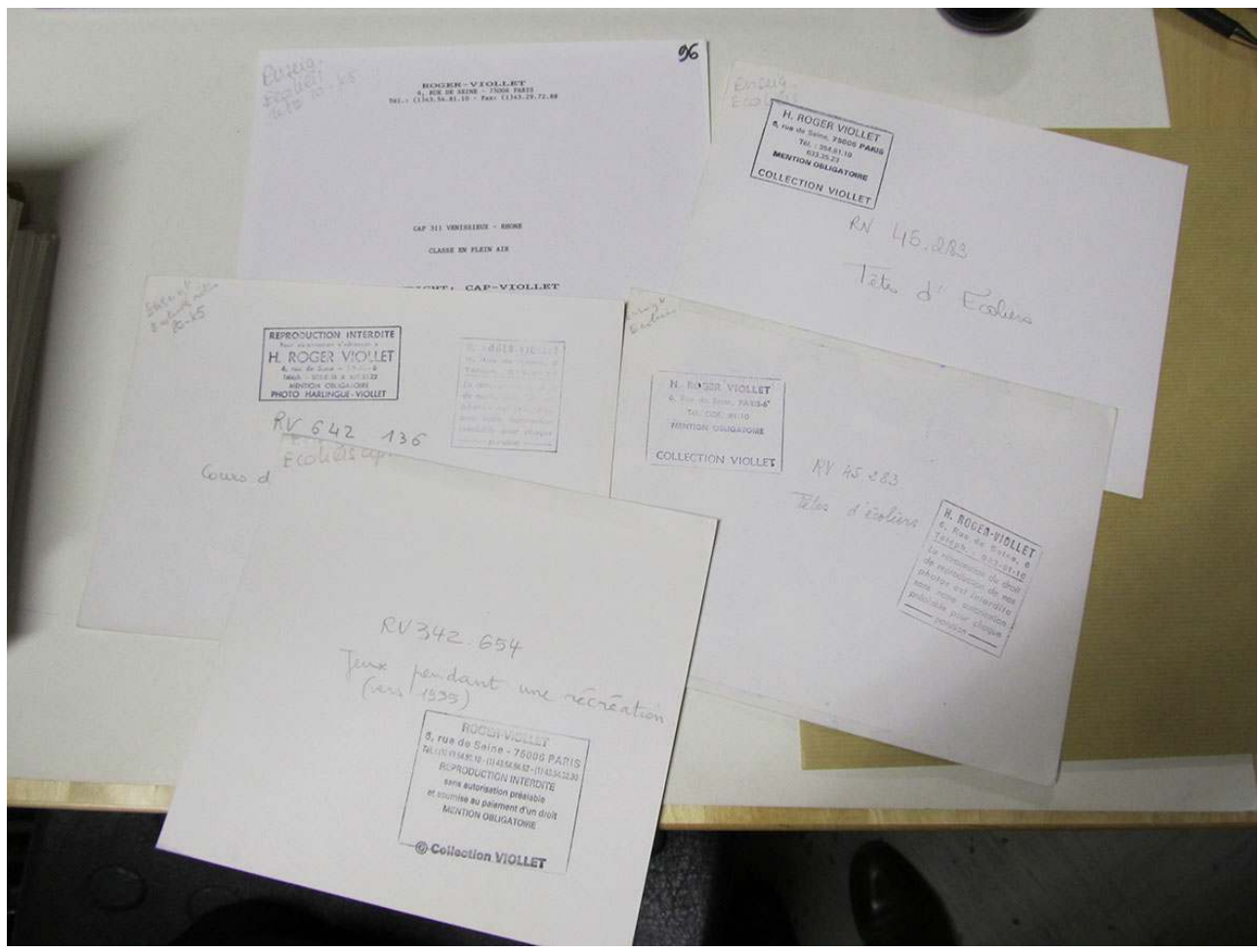

Dos de tirages de presse prêtés et rendus, 2014

(c) Denis Fruit / Parisienne de Photographie.

21 La manière de travailler était traditionnelle : consultation et choix des photographies, établissement d'un bordereau de prêt portant numéro du négatif et légende abrégée, retour des tirages prêtés non utilisés dans les délais impartis (sinon relance car un nonretour pouvait signifier qu'il faudrait procéder à un retirage pour que la photographie reste disponible en boîte), rangement des tirages prêtés et retournés non utilisés, retour des tirages utilisés et facturation des droits de reproduction, rangement des tirages utilisés (fig. 16). 
Figure 16

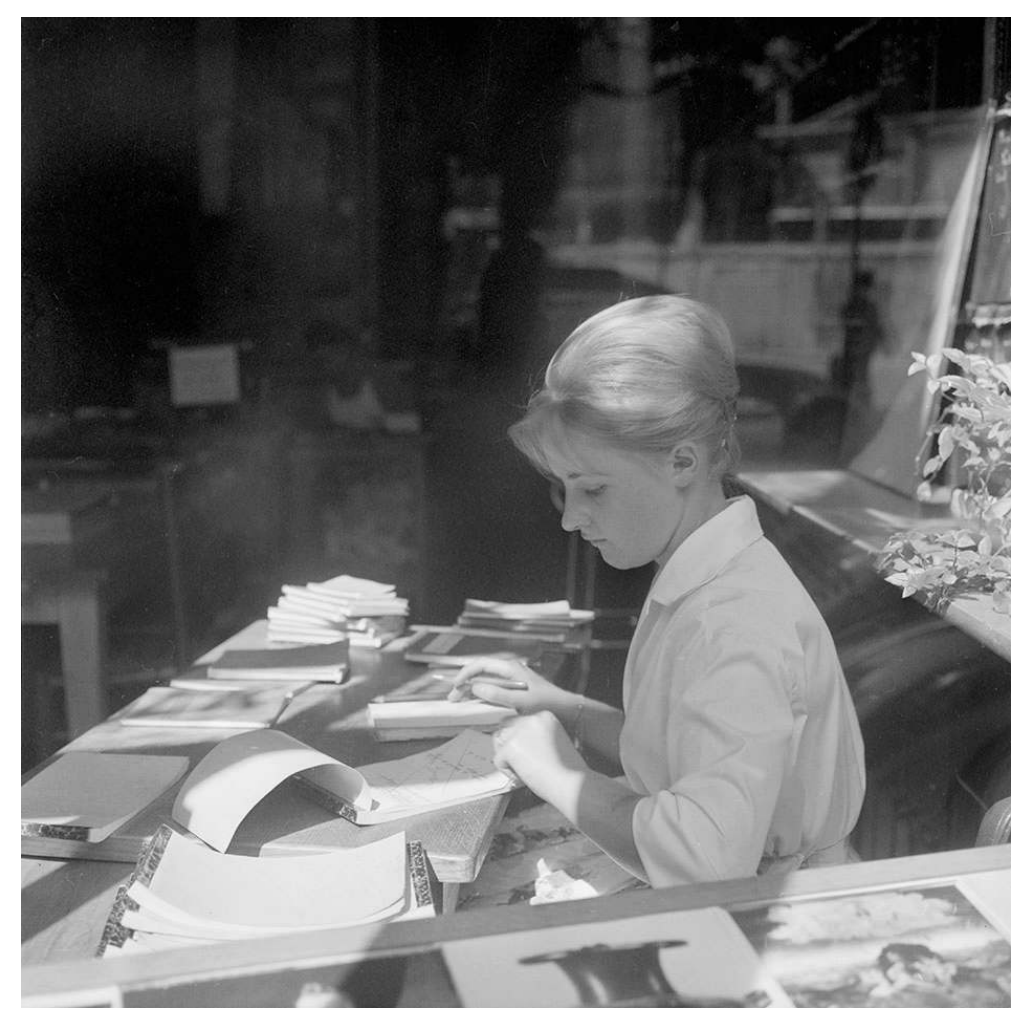

Documentaliste pointant les retirages. Roger-Viollet, 6 rue de Seine, septembre 1960.

(c) Anonyme / Roger-Viollet.

Ce que l'on nomme dans le jargon, les «CGV », les fameuses «conditions générales de vente ", étaient en effet drastiques. Inscrites en tout petits caractères au dos des bordereaux de communication ${ }^{7}$ et signées obligatoirement par tous les nouveaux clients et à chacune de leurs commandes par les clients « farceurs » (ceux-ci dûment signalés par les documentalistes historiques aux nouveaux, lesquels se voyaient chargés de la chasse au mauvais payeur.

23 Ces conditions générales de vente sont la réponse claire au statut des photographies d'agence et de photographes figurant encore dans les fonds des journaux, éditeurs et autres utilisateurs de photographies: «droit de garde: les photographies sont communiquées à titre de prêt pour une période déterminée, voir au recto ${ }^{8}$, passé ce délai, un droit de garde sera perçu, voir bas de page, au-delà de 2 mois de retard, les photographies seront considérées comme perdues et une indemnité de perte sera facturée ».

Identiques jusqu'en $2005^{9}$, ces conditions ont été revues en 2006 et 2012 pour s'adapter au marché numérique (mais toujours imprimées en police de caractères 6 !) :

La transmission des images aux cessionnaires sous quelque forme que ce soit n'implique pas l'autorisation d'utilisation de reproduction, de représentation ou d'inspiration. La transmission pourra faire l'objet d'une facturation de frais techniques indépendamment des droits d'exploitation d'images sauf accord préalable écrit de Roger-Viollet ou de la Parisienne de Photographie. Le cessionnaire s'interdit formellement de constituer une base de données physique ou numérique à partir des images transmises par Roger-Viollet. Le cessionnaire n'est pas autorisé à conserver en archive sous quelque support que ce soit, les 
images transmises par Roger-Viollet ou à télécharger sur le site internet de RogerViollet au-delà de 7 jours à compter de leur utilisation effective. Le cessionnaire s'engage en conséquence à détruire les fichiers numériques ou le cas échéant à retourner tout document argentique ou numérique, sélection d'images sur CD comprises appartenant à Roger-Viollet dès l'expiration de ce délai. En outre, est obligatoire de faire figurer le crédit photographique et la source du fichier pendant son stockage temporaire par le cessionnaire ${ }^{10}$.

En effet, la politique de numérisation intensive menée à partir de 1998 avait rendu rare dès l'année 2000 la communication de tirages.

\section{Postérité des Collections Roger-Viollet}

Menée avec une efficacité redoutable, la numérisation fera un temps de Roger-Viollet un précurseur parmi les agences historiques proposant des fichiers. Était numérisé tout ce que les clients empruntaient, publié ou non, ainsi que ce que l'on nomme les " marronniers", les anniversaires, les grands et les moyens sujets, les « niches » (fig. 17). En 1998, on avait été jusqu'à « enterrer » toutes les personnalités âgées de plus de 70 ans afin d'avoir sous la main les tirages nécessaires à leur nécrologie... Peu délicat sans aucun doute, mais commun et efficace. Le tout en veillant à mettre en diffusion ce qui était clarifié et exploitable du point de vue du droit ${ }^{11}$.

Figure 17

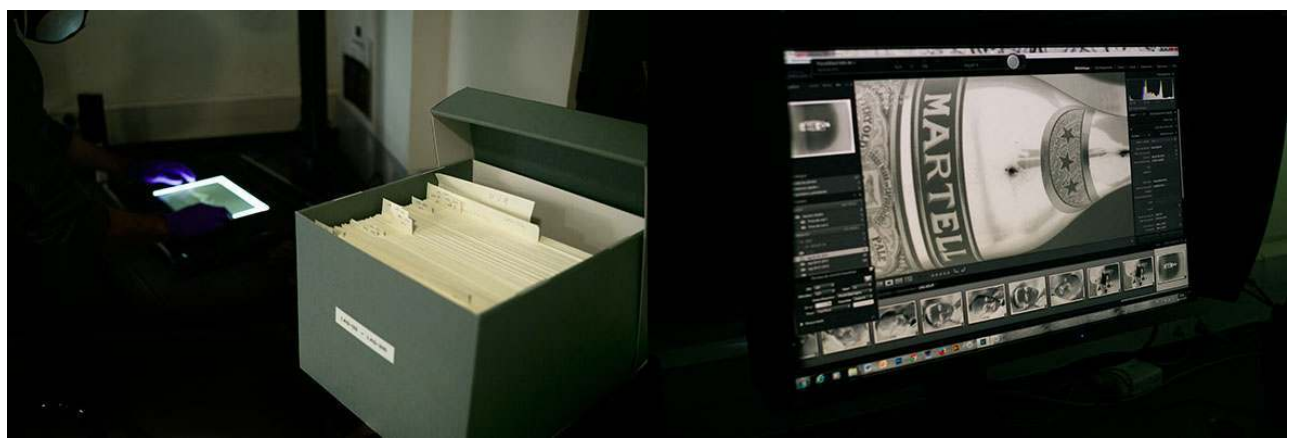

Numérisation des supports en nitrate de cellulose du fonds Laure Albin Guillot dans les réserves rue Dubrunfaut. Paris, 2 février 2015.

(c) Denis Fruit / Parisienne de Photographie.

Le principe des tirages de presse est donc qu'ils soient prêtés et non donnés. Il y eut évidemment une tolérance et une confiance à l'égard des clients fiables qui conservaient des tirages à des fins de réutilisation pour s'économiser de nouvelles recherches, et ce, de très longues années, voire des décennies. Des clients qui, un beau jour, trient (souvent parce qu'ils déménagent) et rendent à chacune des agences ce qui leur revient : RogerViollet a reçu ainsi plusieurs cartons provenant de L'Express il y a une quinzaine d'années et plus récemment, du Parisien.

D'autres ensembles iconographiques constitués à partir de fonds d'agences posent question, comme l'iconothèque des éditions Flammarion, versée à l'Institut de la mémoire de l'édition contemporaine (IMEC) et qui contient bon nombre de tirages Roger-Viollet dont le retour avait été envisagé en même temps qu'avait été proposé l'achat de cette même iconothèque qui contenait des tirages qui auraient dû être rendus à l'agence depuis parfois des lustres... Il est évident que le fonds iconographique d'une maison d'édition est 
un élément archivistique passionnant en soi, mais des solutions juridiques peuvent être trouvées, des dépôts par exemple, qui laisseraient son intégrité à l'archive constituée en laissant à chacun la propriété de son bien, comme cela est en cours pour le fonds iconographique de la collection "Terre humaine ", constitué par Jean Malaurie pour les éditions Plon et entré à la Bibliothèque nationale de France.

En ce sens, qu'il s'agisse de procéder après vingt ans d'exercice à un retour d'expérience, d'analyser chacun des fonds par auteur ou la constitution même des Collections RogerViollet, d'étudier et de témoigner de ce qui fut le quotidien agité et fragile des agences photographiques dans la seconde moitié $\mathrm{du} \mathrm{xx}^{\mathrm{e}}$ siècle, d'analyser historiquement et plus vastement l'évolution et la disparition des dites agences... qu'il s'agisse de penser en termes historiques ou juridiques ${ }^{12}$ ou de monter un plan scientifique et culturel, on imagine aisément que conserver le plus possible d'archives en tous genres est déterminant.

Les archives afférentes aux acquisitions et aux traitements des fonds sont conservées usuellement puisqu'elles accompagnent le patrimoine et sa conservation ${ }^{13}$.

Pour autant, le contexte d'une agence n'a rien à voir avec la constitution d'un département photographique muséal. Chez un auteur et a fortiori dans une agence, les photographies viennent par " grappe » (par auteurs, par reportages, par sujets, par types de support, etc.) et non à l'unité ou comme des œuvres finies et « exposables ».

Le quotidien, la vie économique autour des photographies, leurs pratiques et leurs usages, leur évolution dans le temps, les contacts entre chacun des acteurs (nombreux et divers) du système, les contrats, les dossiers clients et les correspondances, les agendas, les cahiers de courriers, les notes, les mémoires, les ouvrages produits et de fait, tout ou partie des justificatifs, ainsi que la décoration choisie pour mettre en valeur la marque et son implantation historique ou a contrario, sa modernité... : tout fait sens.

Et seul le tout permet de saisir les tenants et aboutissants de cette industrie lucrative que furent les agences.

Il faudrait, pour faire passer à la postérité ce que furent les agences, en conserver la lisibilité, donc conserver dans leur intégralité et leur intégrité ces pièces si diverses dans un ordre respectant ce qui fut.

On déplore trop souvent un «effet Orangina » lors des transferts de collections amples, lequel effet altère pour toujours la lisibilité que pourront avoir de ce passé ceux qui ne l'auront pas connu.

36 En effet, et pour ne prendre qu'un exemple, les liens entre la production d'une photographie, son développement, son tirage, sa mise en diffusion, sa communication et son retour pour pige et facturation des droits d'exploitation, liens qui nous paraissaient évidents il y a moins de vingt ans, sont inconnus de la génération tout numérique. Et on le sait, restituer la notion de lien sans éléments témoins est une gageure (fig. 18, 19, 20). 
Figure 18

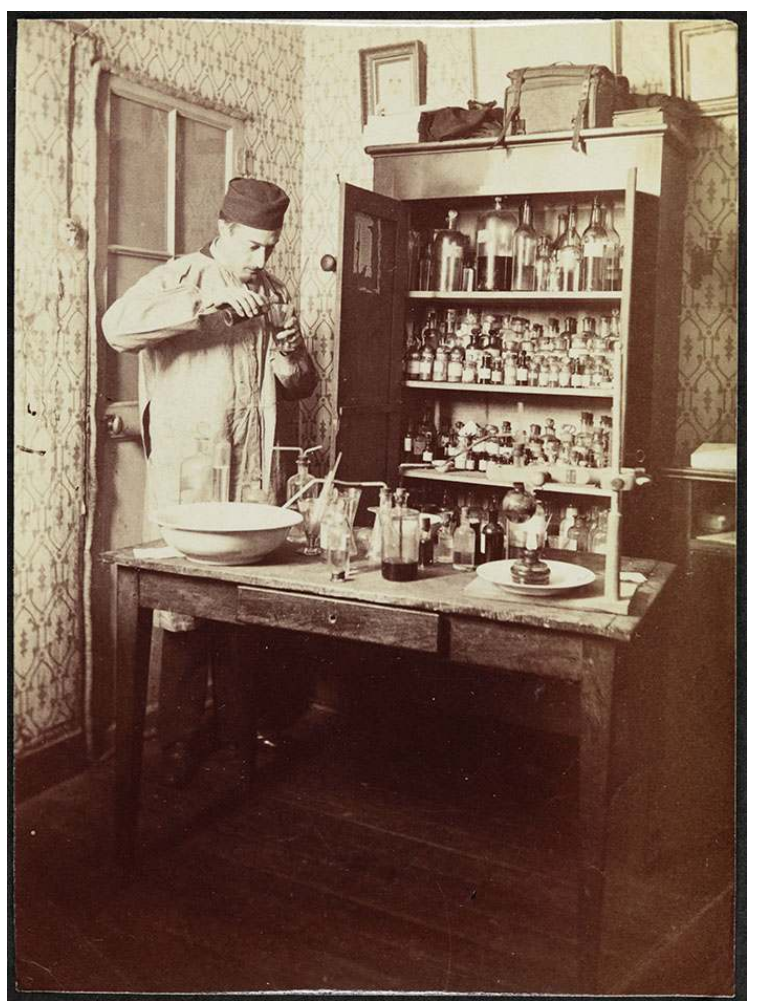

Henri Roger dans son laboratoire. Paris, 24 rue Servandoni, 24 juin 1892.

(c) Henri Roger / Roger-Viollet. 
Figure 19

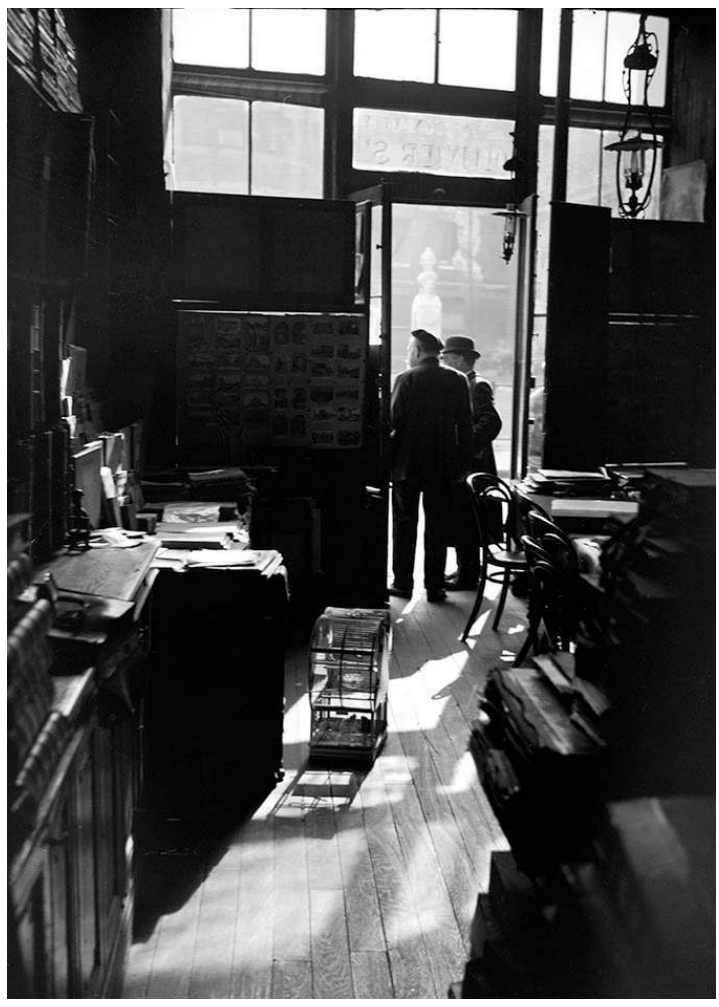

LAURENT OLLIVIER SUR LE PAS DE PORTE dU 6 RUE DE SEINE, AVRIL 1938.

(c) Hélène Roger-Jean Fischer / Roger-Viollet. 


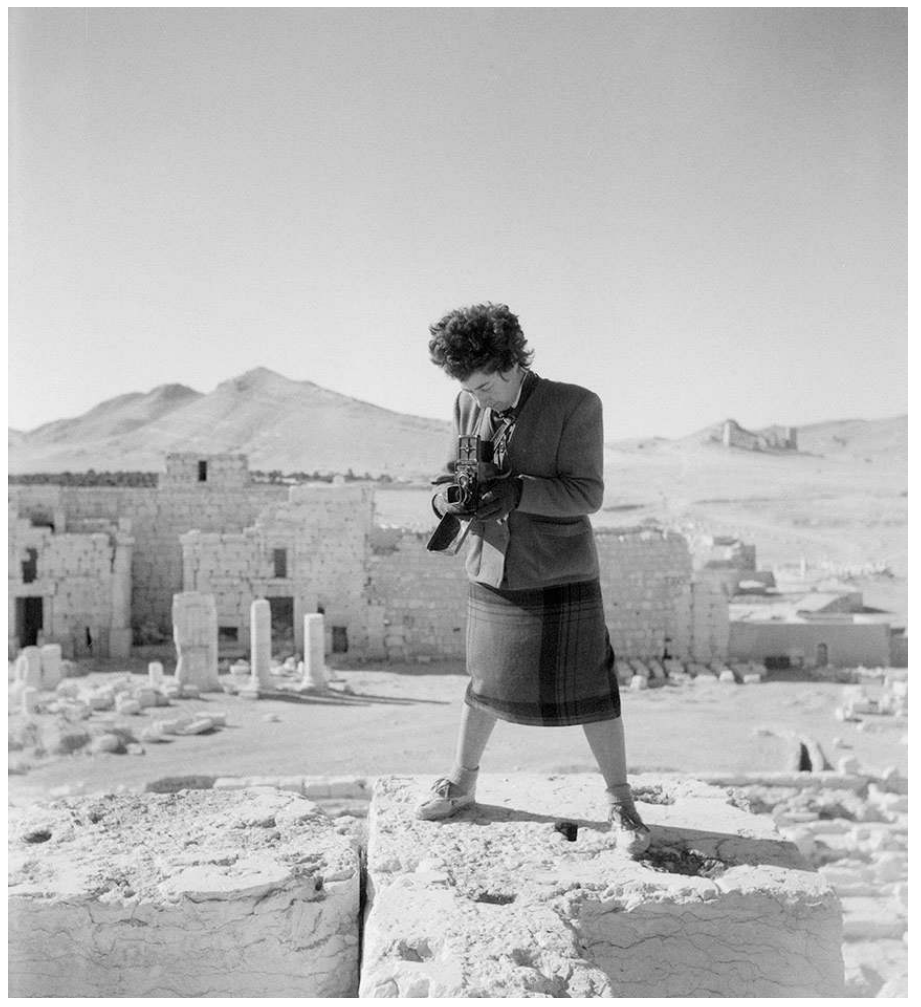

hélène ROger dANS LE TEMPLE de BAAL, PALMYRE, SYRIE, NOVEMBRE 1953.

(C) Jean Fischer / Roger-Viollet.

Bien entendu et pour des raisons de place, cette idée semble toujours exagérée voire délirante. Pourtant, c'est tout ce que l'on aura su conserver de ce qui fut la gestion réelle d'une agence photographique qui permettra d'arriver à la compréhension de ce que fut la photographie et son marché au $\mathrm{xx}^{\mathrm{e}}$ siècle.

\section{NOTES}

1. - Respectivement 1901-1985 et 1904-1985.

2. - 1866-vers 1925.

3. - Les fonds Henri et Ernest Roger sont conservés dans les Collections Roger-Viollet alors que le fonds Henry Viollet a été légué par ses soins à l'État et est aujourd'hui conservé à la Bibliothèque universitaire des langues et civilisations (Bulac).

4. - Identifiés au fur et à mesure des inventaires par l'équipe en charge des Collections, ces supports sont dotés d'un crédit spécifique selon leur provenance.

5. - Il est à noter que depuis 2002, des campagnes de conservation grandes et petites se succèdent au sein des Collections Roger-Viollet : épuisement des reliquats, reconditionnement, séparation des types de supports, isolement des supports en nitrate de cellulose, dépoussiérage et 
stabilisation des plaques de verre grand format, traitement des albums photographiques anciens et des photographies enchâssées, etc.

6. - Un test de culture générale "taquin » présidait au choix des nouveaux, et gare à qui ne connaissait pas ses citations politiques ou une certaine petite ville portuaire algérienne !

7. - Par exemple, la perte d'un Ektachrome était facturée 2000 francs.

8. - L'usage était d'accorder un mois au client, davantage s'il s'agissait d'un projet d'édition à long terme.

9. - En 2005, est créée la Parisienne de Photographie, société anonyme d'économie mixte locale (Saeml) qui répond à l'appel d'offre de la Ville de Paris pour conserver les Collections RogerViollet, numériser et diffuser le patrimoine iconographique et photographique parisien.

10. - Il est à noter que lors du lancement de la politique de numérisation, en 1998, l'agence RogerViollet avait fait le choix de ne plus ou presque plus produire de tirages mais d'envoyer des fichiers numériques.

11. - Il est à noter que les fichiers ainsi générés, même si le temps requiert qu'une partie d'entre eux soient refaits, représentent à la fois une "force d'exploitation commerciale » et un patrimoine secondaire réel et tangible. Réel car il permet de ne pas recourir aux œuvres originales, souvent fragiles, tangible car consultable et dont il faut assurer la pérennité par des vérifications et des migrations.

12. - Ce que l'on omet hélas très souvent quand il s'agit de photographie alors qu'il s'agit du cœur agissant du médium dès que l'on parle commercialisation.

13. - Qui plus est dans le cas qui nous occupe, un patrimoine devenu public et dont l'étude durera sans aucun doute plus que les moins de 70 ans d'exercice réel de l'agence Roger-Viollet. Surtout si on y ajoute l'analyse de ce qui n'a pas fait l'objet de retours et perdure dans les fonds de périodiques, de maisons d'édition et de tout autre client.

\section{RÉSUMÉS}

Fondé en 1938 par Hélène Roger et Jean Fischer, Roger-Viollet s'installe chez Laurent Ollivier, «marchand d'images» connu depuis 1880. L'emplacement répond à son environnement où se côtoient étudiants, artistes, revues et maisons d'édition, autant de clients potentiels. Fermée pendant la Seconde Guerre mondiale, la boutique rouvre dès 1944 et le couple se lance dans les achats: fonds de studios photographiques, de photographes professionnels, amateurs, périodiques illustrés, arts graphiques, bibliophilie... le but des fondateurs est de réunir de quoi répondre à tout besoin iconographique. En digne fille d'inventeur, Hélène Roger est sensible à la propriété intellectuelle. Aussi est-il décidé de prêter les tirages et de ne céder que des droits de reproduction en fonction des utilisations qu'en font les clients. Classées sans recours à un thésaurus mais suivant une logique intellectuelle et commerciale imparable, les collections évoluent de manière organique et en suivant les demandes et évolutions de la profession d'iconographe; menée avec une efficacité redoutable, la numérisation fera un temps de RogerViollet un précurseur parmi les agences historiques. Pour faire passer à la postérité et pouvoir étudier ce que furent les agences, il est indispensable de conserver dans leur intégrité des pièces très diverses dans un ordre respectant ce qui fut: vie économique, pratiques et usages, évolutions, contacts et contrats entre les acteurs du système, ouvrages produits et justificatifs... tout fait sens, et seul le tout permet de saisir les tenants et aboutissants de cette industrie qui fut lucrative. 
Roger-Viollet is a well-known French photo agency that was founded in 1938 by Hélène Roger and Jean Fischer. The agency set up in the premises of Laurent Ollivier, an 'image merchant' who was well known from the 1880s on. The sixth-arrondissement address in Paris placed it in an environment close to its potential clients amongst students, artists, magazines, publishing houses and so on. The agency was closed during the Second World War but opened again in 1944, when the couple undertook a purchasing policy: collections of photo agencies, of professional or amateur photographers, illustrated magazines, graphic arts, rare books... Their aim was to be able to reply to the needs of any picture researcher. As the daughter of her photographer-father, Hélène Roger was particularly sensitive to questions to do with copyright. She consequently decided that the photographic prints would be lent to clients and only the reproduction rights would be sold, the prices fixed according to the usage and diffusion of the picture. The photos were organised without the use of a thesaurus but in an efficient way that was inspired by intellectual and commercial logics. The collections evolved in an organic way, following the changing requirements of professional picture researchers. For a time, the Roger-Viollet agency was clearly a precursor amongst such agencies on account of the digitisation of its images, undertaken with remarkable efficiency. In order to understand such agencies and to guarantee their lasting usefulness, it is vital to conserve all the archival elements, however varied, respecting the order of what constituted the agency's economic activity, its practices and usages, its changes over time, its contacts and contracts with other actors in the field, the works produced, their supporting documents... Anything can contribute to understanding the whole picture and seeing what made this activity such lucrative business.

\section{INDEX}

Mots-clés : Roger-Viollet, production, tirage, négatif, reportage, acquisition, étude, légende, classement, intégration, conservation, thésaurus, reproduction, iconographique, marché, numérisation

Keywords : Roger-Viollet, production, print, negative, reportage, acquisition, study, caption, classification, integration, conservation, thesaurus, reproduction, iconographical, market, digitisation

\section{AUTEUR}

\section{DELPHINE DESVEAUX}

Directrice des Collections Roger-Viollet, Bibliothèque historique de la Ville de Paris

delphine.desveaux@paris.fr 